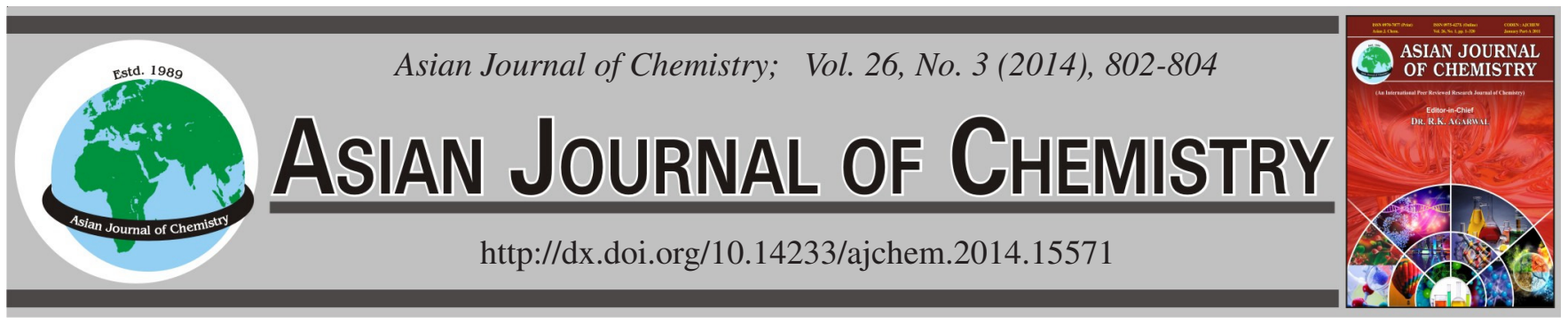

\title{
Depressant Action of Alcohol Extract of Jasmine Root on Central Nervous System in Mice
}

\author{
Tian Ning ${ }^{1}$, Hong Liang ${ }^{1}$, Wen-Zhen Huang ${ }^{2}$, Xue-Lin Duan ${ }^{3}$, Yue Peng ${ }^{3}$ and Tie-Jian Zhao ${ }^{3, *}$
}

\author{
${ }^{1}$ Department of Pharmacology, Guangxi Health Vocational \& Technical College, Nanning 530023, P.R. China \\ ${ }^{2}$ Guangxi Maternal and Child Health Hospital, Nanning 530003, P.R. China \\ ${ }^{3}$ Basic Medical College, Guangxi University of TCM, Nanning 530001, P.R. China
}

*Corresponding author: Tel: +86 13014989836; E-mail: 13014989836@163.com

Received: 3 April 2013;

Accepted: 6 August 2013;

Published online: 30 January 2014;

AJC-14641

The pharmacological effects on the central nervous system of the alcoholic extract of jasmine root were explored to elucidate the mechanism and provide experimental basis for the development and application of jasmine root. The independent activity determination method is applied to observe the drug sedation action and sodium pentobarbital synergy is taken to observe its hypnotic effect. Hot plate method and acetic acid-induced writhing method are used to observe the analgesic action. Alcohol extract of jasmine root decreased the independent activity of mice, which may indicated its sedative hypnotic effect. The synergistic effect with pentobarbital sodium may indicated its hypnotic effect. Alcoholic extract of jasmine root can reduce acetic acid-induced writhing times and increase pain threshold in mice, indicating its analgesic action. Alcoholic extract of jasmine root have sedative-hypnotic effect and the effect of analgesia in mice.

Keywords: Jasmine root, Sedative hypnotic effect, Analgesic action.

\section{INTRODUCTION}

Jasmine root (Radix J. asmini Sambac RJS) is the root of the plant jasmine Oleaceae. Five compounds were isolated and identified from jasmine root though chloroform fraction and butanol fraction of the alcohol extract, two of them were lignan compounds isolated from Jasminum for the first time ${ }^{1}$ According to the literature, lignan compounds performed a depressant action of the central nervous system ${ }^{2}$. It was also reported that oral solution of jasmine root can cure insomnia ${ }^{3}$. For the further study, we focused on the central nervous system inhibition by alcohol extract of jasmine root.

\section{EXPERIMENTAL}

The SPF mice (purchased from Experimental Animal Center in Guangxi University of TCM, weighing 18-22 g), alcohol extract of jasmine root (provided by Department of Basic Medical, Guangxi University of TCM,) Rotundine injection (Guangxi Nanning Baihui Pharmaceutical factory, Production batch: 1105001), Nikethamide injection (Shanghai modern Hasson Pharmaceutical production, Production batch: 11060112), Acetic acid (Tianjin reagent Factory produced), Pentobarbital sodium (Shanghai modern Hasson Pharmaceutical production, production batch: 1107008), Smart Hot Plate Instrument (Chengdu instrument Factory, RB-200), Biological and Functional Experimental System (Chengdu instrument Factory, BL-420E).
Jasmine root stock solution was concentrated to $5 \mathrm{~g}$ crude drug per $\mathrm{mL}$. Refer to the result LD50 $=89.95 \mathrm{~g} / \mathrm{kg}$ weight, the mice in treatment groups were treated by garage with the concentrated solution at the dosage of $18.8 \mathrm{~g} / \mathrm{kg}, 12 \mathrm{~g} / \mathrm{kg}$, $9 \mathrm{~g} / \mathrm{kg}$ body weight respectively.

Locomotor activity: Improved sensitive locomotor activity measuring device ${ }^{4}$ was applied (BL-420 E biological and functional experimental system connected with tension transducer). 48 mice, of half male, was divided into four groups randomly, $18.8 \mathrm{~g} / \mathrm{kg}, 12.0 \mathrm{~g} / \mathrm{kg}, 9.0 \mathrm{~g} / \mathrm{kg}$ of concentrated solution groups and the saline group, 12 in each group. All the mice were administrationed by garage $0.05 \mathrm{~h}$ before intraperitoneal injection of $2 \%$ nikethamide $0.1 \mathrm{~mL} / 10 \mathrm{~g}$ and selfactivity curve recording within $10 \mathrm{~min}$ each for four mice.

Synergy with pentobarbital sodium: 40 mice, of half male, were divided into four groups randomly as described above $(n=10)$. Each mice was given a intraperitoneal injection of sodium pentobarbital $(0.2 \mathrm{mg} / 10 \mathrm{~g}) 0.5 \mathrm{~h}$ after administration. The loss of righting reflex was recorded as the occurrence of sleep

Pain threshold in hot-plate test ${ }^{5}$ : Male mouse were selected and placed on a hot plate (Smart RB-200 hot plate meter) of $55 \pm 0.5^{\circ} \mathrm{C}$ individually and the time from plate-contacting to hind foot-licking was recorded as pain threshold. The mice whose pain threshold in 5-30 s were seen qualified, excluding those less than $5 \mathrm{~s}$ or more than $30 \mathrm{~s}$. Pain threshold were 
measured twice before administration, at intervals of $5 \mathrm{~min}$ and their average was caculated as basic pain threshold. 40 qualified mice were divided into four groups $(n=10)$ randomly: high, medium, low dose groups of alcoholic extract of jasmine root as described above and rotundine injection as positive control group. Pain threshold of each mice was measured at $30 \mathrm{~min}, 45 \mathrm{~min}$ and $60 \mathrm{~min}$ after intragastric administration. 60 s was recorded for those beyond $60 \mathrm{~s}$ without responding.

Acetic acid-induced writhing in mice $^{6}: 50$ mice, of half male, were randomly divided into five groups $(n=10)$ : high, medium, low doses groups of alcoholic extract of jasmine root and rotundine injection as positive control group and saline as negative control group. All the mice were given intraperitoneal injection of $0.6 \%$ acetic acid $0.1 \mathrm{~mL} / 10 \mathrm{~g}, 30 \mathrm{~min}$ after intragastric administration. The number of writhing was measured respectively.

Detection method: Hot plate licking extended percentage $=($ threshold after administration- the basic threshold $) /$ the basic threshold $\times 100 \%$. Writhing inhibition percentage $=$ (control group writhing times - administration group writhing times) / control group writhing times $\times 100 \%$. Statistical analysis was performed with the SPSS 13.0. All data were expressed as means $( \pm$ SEM). Statistical analyses were performed with either a Student t-test or an one-way analysis of variance (ANOVA). A p $<0.05$ was considered statistically significant.

\section{RESULTS AND DISCUSSION}

Locomotor activity: As shown in Fig. 1, the three dosages of alcoholic extract of jasmine root (AEJR) reduced the spontaneous activity, inhibiting the exercise-induced agitation of nikethamide, significantly.

Synergy with pentobarbital sodium: Before the mice get into sleep, mostly performing humpback and stagger. High, medium and low dose groups of alcoholic extract of jasmine root could reduce disappearance time of righting reflex induced by sodium pentobarbital. Compared to the control group, pure sodium pentobarbital group, high dose group had a significant variation, $\mathrm{P}<0.05$, while the low, middle dosages groups had no significant variation( the low-dose group $\mathrm{P}=0.315$, middle dose group, $\mathrm{P}=0.105)$. The results are shown in Table-1.

\begin{tabular}{lcc}
\multicolumn{3}{c}{ TABLE-1 } \\
\multicolumn{2}{c}{ SYNERGY EFFECT OF ALCOHOLIC EXTRACT OF } \\
JASMINE ROOT WITH PENTOBARBITAL SODIUM \\
\hline \multicolumn{1}{c}{ Groups } & $\begin{array}{c}\text { Disappearance time of } \\
\text { righting reflex }\end{array}$ & T value \\
\hline Sodium pentobarbital & $344.5 \pm 163.7$ & \\
Low dose group & $368.3 \pm 153.9$ & 0.315 \\
Medium dose group & $291.2 \pm 113.3$ & 0.105 \\
High dose group & $257.9 \pm 114.5$ & 0.033 \\
\hline All data are expressed as the means \pm SEM &
\end{tabular}

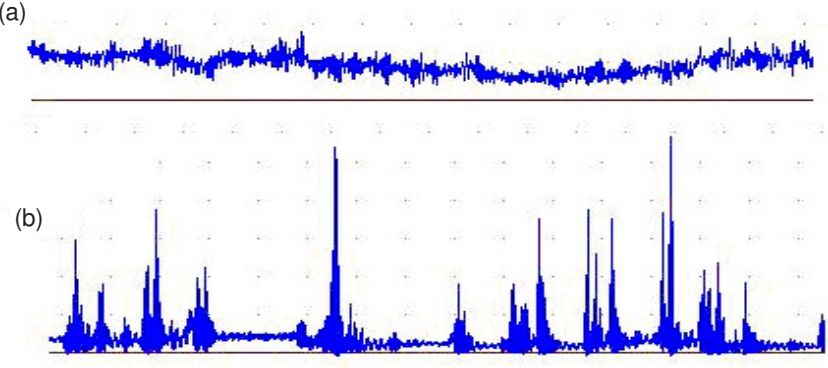

(c)

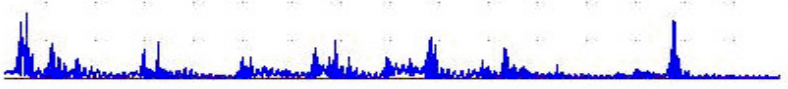

(d)

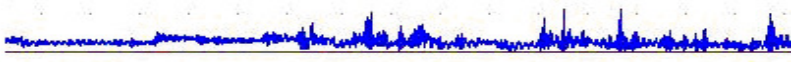

(e)

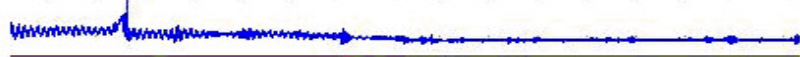

Fig. 1. Effect of different doses of AEJR and Nikethamide on spontaneous activity (a) Saline (b) Nikethamide (c) Low-dose of AEJR (d) Medium-dose of AEJR (e) High-dose of AEJR. All data are expressed as the means \pm SEM.

Hot plate method $^{5}$ in mice foot pain reaction pain threshold in hot-plate test: Hot plate pain threshold of mice in each group had no variation before administration. Compared with those before administration, low-dose group of alcoholic extract of jasmine root at $0.5 \mathrm{~h}$ had no difference $(\mathrm{P}=0.077)$, while a significant enhancement at 45 and $60 \min (\mathrm{P}<0.05)$. Compared with the positive control group, medium-group and high-dose group both showed a inferior increase, though showed a significant enhancement $(\mathrm{P}<0.05)$ at 30, 45, 60 min after administration compared with those before administration. The results of hot plate licking extended percentage are shown in Table- 2 .

Acetic acid-induced writhing in mice: Compared with the saline negative control group, low, medium and high dose groups of alcoholic extract of jasmine root were significantly reduced the number of writhing in mice in each group $(\mathrm{P}<$ $0.05)$, though the decrease of the number was inferior to rotundine. Inhibition percentage of writhing of low-dose group, medium-dose group, high-dose group and Rotundine positive control group were, 50.03, 70.78, 57.79 and $87.76 \%$, respectively. The results are shown in Table-3.

Jasmine root is bitter, warm and toxic. It is well applied to the treatment of bruises, dental caries, headache and insomnia embolism for its analgesic effect ${ }^{7}$. Our present data shows that alcoholic extract of jasmine root decreases inde-

TABLE-2

EFFECT OF AEJR ON HOT PLATE PAIN THRESHOLD IN MICE

\begin{tabular}{lccccccc}
\hline \multirow{2}{*}{ Groups } & $\begin{array}{c}\text { Basic pain } \\
\text { threshold }\end{array}$ & \multicolumn{2}{c}{ Basic pain threshold after adminnistration } & \multicolumn{2}{c}{ Pain threshold extended percentage } \\
\cline { 3 - 8 } & $14.06 \pm 4.64$ & $17.73 \pm 5.49$ & $24.21 \pm 12.91$ & $18.66 \pm 4.28$ & $30.00 \%$ & 85 min & 60 min \\
\hline Low dose & $14.15 \%$ & $61.87 \%$ \\
Mediumdose & $18.25 \pm 6.86$ & $24.83 \pm 10.33$ & $25.19 \pm 10.33$ & $21.04 \pm 8.16$ & $36.03 \%$ & $38.56 \%$ & $19.70 \%$ \\
High dose & $19.14 \pm 3.74$ & $28.11 \pm 11.55$ & $22.76 \pm 12.74$ & $27.97 \pm 16.33$ & $46.84 \%$ & $8.89 \%$ & $46.11 \%$ \\
Rotundine & $19.26 \pm 6.67$ & $60.00 \pm 0.00$ & $60.00 \pm 0.00$ & $60.00 \pm 0.00$ & $100.00 \%$ & $100.00 \%$ & $100.00 \%$ \\
\hline
\end{tabular}

All data are expressed as the means \pm SEM 


\begin{tabular}{lcc}
\hline \multicolumn{3}{c}{ TABLE-3 } \\
\multicolumn{3}{c}{ EFFECT OF ALCOHOLIC EXTRACT OF JASMINE ROOT } \\
ON ACETIC ACID-INDUCED WRITHING IN MICE
\end{tabular}

pendent activity induced by nikethamide, which indicating its sedative hypnotic effect. And it has synergistic effect with pentobarbital sodium, indicating its hypnotic effect. On the other hand, it can reduce torsion times and significantly increase the pain threshold of mice, indicating its analgesic action. writhing inhibition percentages are more than $50.0 \%$ in acetic acid induced writhing experiment. All the study shows the inhibitory effect on the central nervous system. Study on the inhibition of CNS always aimed at traditional Chinese medicine of sedative and epilepsy and seldom publicly reported for the research and application of jasmine root. Further research on the mechanism and chemical structure of the active components is needed. This study would give a animal experimental foundation for the further research and clinical application of jasmine root.

\section{Conclusion}

Alcoholic extract of jasmine root have sedative-hypnotic effect and the effect of analgesia in mice.

\section{ACKNOWLEDGEMENTS}

The acknowledgement is extendend to the National Natural Science Foundation of China (Nos. 81160553 and 81260675 ) and the Natural Science Foundation of Guangxi University of TCM (2009 Guangxi KeJi word 3, 92-1) for the financial supports.

\section{REFERENCES}

1. Y. Zhang and Y.M. Zhao, Med. J. Chinese People's Liberation Army, 22, 279 (2006).

2. X.S.Yao, Natural Medicinal Chemistry, People's Health Publishing House, Beijing, p. 140 (2001).

3. X. Ning and S.H. Zhou, Integr. Tradit. Chinese Western Med. China, 24, 476 (2004).

4. J.Q. Bao, J.M. Feng and Y.Y. Sun, Chinese Pharmacol. Bull., 10, 155 (1994).

5. S.Y. Xu, R.L. Bian and X. Chen, Pharmacological Experiments Methodology, People's Health Publishing House: Beijing, pp. 882-888 (2001).

6. Q. Chen, Pharmacology Research Methodology of Traditional Chinese Medicine, People's Health Publishing House: Beijing, p.361 (1996).

7. T.K. Huang, Compendium of Materia Medica, Chinese Journal of Pharmaceuticals Publishing House: Beijing, p. 1472 (2000). 